\title{
Review on Thermoelectric Refrigeration: Materials and Technology
}

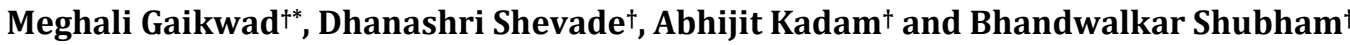 \\ †Meghali Gaikwad, Mechanical Engineering, MITCOE. Pune University, India \\ Accepted 02 March 2016, Available online 15 March 2016, Special Issue-4 (March 2016)
}

\begin{abstract}
Conventional refrigeration systems use Chloro Fluoro Carbons (CFCs) and Hydro Chlorofluorocarbons (HCFCs) as heat carrier fluids. Use of such fluids in conventional refrigeration systems has a great concern of environmental degradation and resulted in extensive research into development of novel Refrigeration and air conditioning technologies. Thermoelectric refrigeration is one of the techniques used for producing refrigeration effect. A brief review about introduction of thermoelectricity, principal of thermoelectric cooling and thermoelectric materials has been presented in this paper. The research and development work carried out by different researchers on development of thermoelectric $R \& A C$ system has been thoroughly reviewed in this paper.
\end{abstract}

Keywords: Thermoelectric module, Peltier effect, Figure of Merit, Device Design Parameter, Seebeck coefficient, Coefficient of performance.

\section{Introduction}

Refrigeration is defined as the process of cooling of bodies or fluids to temperatures lower than those available in the surroundings at a particular time and place. Thermoelectric refrigeration is one of the techniques used for producing refrigeration effect. Thermoelectric are based on the Peltier Effect. The Peltier Effect is one of the thermoelectric effects; the other two are known as the Seebeck Effect and Thomson Effect whereas the last two effects act on a single conductor, the Peltier Effect is a typical junction phenomenon. There are many products using thermoelectric coolers, including CCD cameras (charge coupled device), laser diodes, microprocessors, blood analyzers and portable and picnic coolers etc. TER is also a good option for food preservation applications \& cooling of pharmaceutical products.

\section{History}

Although commercial thermoelectric modules were not available until almost 1960, the physical principles upon which modern thermoelectric coolers are based actually date back tothe early 1800s. The first important discovery relating to thermoelectricity occurred in 1821 when German scientist Thomas Seebeck found that an electric current would flow continuously in a closed circuit made up of two dissimilar metals, provided that the junctions of the metals were maintained at two different temperatures.

*Corresponding author: Meghali Gaikwad
Seebeck did not actually comprehend the scientific basis for his discovery, however and falsely assumed that flowing heat produced the sameeffect as flowing electric current.

In 1834, a French watchmaker and part-time physicist, Jean Peltier, while investigating the Seebeck Effect, found that there was an opposite phenomenon where by thermal energy could be absorbed at one dissimilar metal junction and discharged at the other junction when an electric current flowed within the closed circuit. Twenty years later, William Thomson (eventually known as Lord Kelvin) issued a comprehensive explanation of the Seebeck and Peltier Effects and described their relationship. At the time, however, these phenomena were still considered to be mere laboratory curiosities and were without practical application.

In the 1930s, Russian scientists began studying some of the earlier thermoelectric work in an effort to construct power generators for use at remote locations throughout their country. This Russian interest in thermoelectricity eventually caught the attention of the rest of the world and inspired the development of practical thermoelectric modules. Today's thermoelectric coolers make use of modern semiconductor technology in which doped semiconductor material takes the place of the dissimilar metals used in early thermoelectric experiments.

The Seebeck, Peltier and Thomson effects, together with several other phenomena, form the basis of functional thermoelectric modules.

In 1909 and 1911 Altenkirch et al. gave the basic theory of thermoelectric. His work explained the 
thermoelectric cooling materials needed to have high Seebeck coefficients, good electrical conductivity to minimize Joule heating, and low thermal conductivity to reduce heat transfer from junctions to junctions. In 1949 Loffe et al developed theory of semiconductors thermo-elements and in1954 Goldsmid and Douglas et al demonstrated that cooling from ordinary ambient temperatures down to below $0^{\circ} \mathrm{C}$ was possible. Rowe et al shortly after the development of practical semiconductors in 1950's, Bismuth Telluride began to be the primary material used in the thermoelectric cooling.

\section{Thermoelectric device principles and theory}

\section{Seebeck Effect}

Suppose there are two conductors of dissimilar metals denoted as material $\mathrm{A}$ and $\mathrm{B}$. The junction temperature at $\mathrm{A}$ is used as a reference and is maintained at a relatively cool temperature $\left(\mathrm{T}_{\mathrm{C}}\right)$.The junction

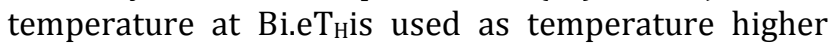
than temperature $T_{C}$. When heat is applied to junction $B$, a voltage $\left(E_{\text {out }}\right)$ will appear across terminals $\mathrm{T} 1$ and $\mathrm{T} 2$ and hence an electric current would flow continuously in this closed circuit. This voltage as shown in Figure known as the Seebeck EMF and it can be expressed as

$\mathrm{E}_{\text {out }}=\alpha\left(\mathrm{T}_{\mathrm{H}}-\mathrm{T}_{\mathrm{C}}\right)$

Where:

- $\alpha=\mathrm{dE} / \mathrm{dT}=\alpha \mathrm{A}-\alpha \mathrm{B}$

- $\alpha$ is the differential Seebeck coefficient or (thermo electric power coefficient) between the two materials, $A$ and $B$, positive when the direction of electric current is same as the direction of thermal current, in volts per ${ }^{\circ} \mathrm{K}$.

- $E_{\text {out }}$ is the output voltage in volts.

- $\mathrm{T}_{\mathrm{H}}$ and $\mathrm{T}_{\mathrm{C}}$ are the hot and cold thermocouple temperatures, respectively, in ${ }^{\circ} \mathrm{K}$.

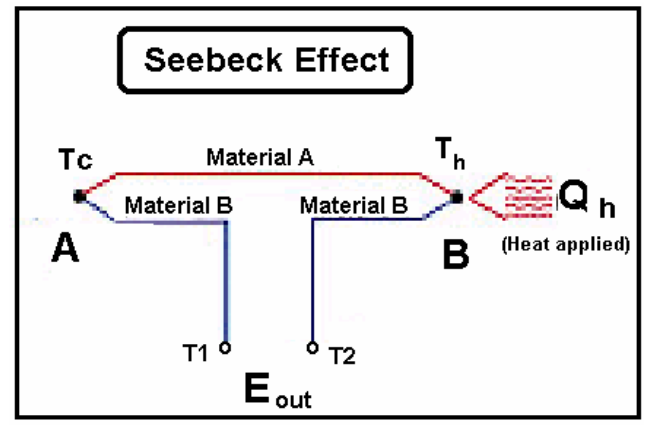

Figure1 Seebeck effect (2)

\section{Peltier Effect}

Later on Peltier found there was an opposite phenomenon to the Seebeck Effect, whereby thermal energy couldbe absorbed at one dissimilar metal junction and discharged at the other junction when an electric current is allowed to flow within the closed circuit.

Figure 2 shows that the thermocouple circuit has been modified to obtain a different configuration that illustrates the Peltier Effect, a phenomenon opposite that of the Seebeck Effect. If a voltage ( $\left.E_{\text {in }}\right)$ is applied to terminals $\mathrm{T}_{1}$ and $\mathrm{T}_{2}$, an electrical current (I) will flow in the circuit. As a result of this, as light cooling effect $\left(Q_{c}\right)$ will occur at thermocouple junction A (where heat is absorbed), and a heating effect $\left(\mathrm{Q}_{\mathrm{H}}\right)$ will occur at junction $B$ (where heat is expelled). This effect may be reversed when a change in the direction of electric current flow will reverse the direction of heat flow. Joule heating, having a magnitude of $\mathrm{I}^{2} \mathrm{x} R$ (where $\mathrm{R}$ is the electrical resistance), also occurs in the conductors as a result of current flow. This Joule heating effect acts in opposition to the Peltier Effect and causesa net reduction of the available cooling. The Peltier effect can be expressed mathematically as

$\mathrm{Q}_{\mathrm{C}}$ or $\mathrm{Q}_{\mathrm{H}}=\beta \times \mathrm{I}$

$=(\alpha \mathrm{T}) \times \mathrm{I}$

\section{Where:}

- $\beta$ is the differential Peltier coefficient between the two materials $A$ and $B$ in volts.

- I is the electric current flow in amperes.

- $Q_{\mathrm{C}}$ and $\mathrm{Q}_{\mathrm{H}}$ are the rates of cooling and heating, respectively, in watts.

Case 1 . When High energy electrons move from right to left, Thermal current and electric current flow in opposite directions and $\beta<0$ i.e Negative Peltier coefficient

Case2.When High energy holes move from left to right, Thermal current and electric current flow in same directions and $\beta>0$ i.e Positive Peltier coefficient

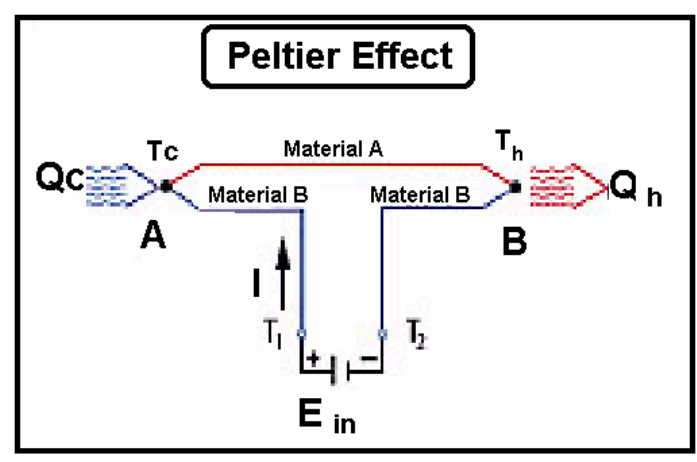

Figure 2 Peltier effect

\section{Thermoelectric Principle of Operation}

Figure 3 shows that the thermoelectric module has been manufactured by using two thin ceramic wafers with a seriesof $\mathrm{P}$ and $\mathrm{N}$ doped bismuth-telluride semiconductor material sandwiched between them. The ceramic material has been there on both sides of thermoelectric which adds rigidity and necessary 
electrical insulation. The $\mathrm{N}$ type material has an excess of electrons, while the $\mathrm{P}$ type material has a deficit of electrons. One $\mathrm{P}$ and one $\mathrm{N}$ make up a couple, as shown in Figure 3. The thermoelectric couples are electrically in series and thermally in parallel. A thermoelectric module can contain one to several hundred couples. As the electrons move from the $\mathrm{P}$ type material to the $\mathrm{N}$ type material through an electrical connector, the electrons jump to a higher energy state absorbing thermal energy (cold side). Continuing through the lattice of material; the electrons flow from the $\mathrm{N}$ type material to theP type material through an electrical connector dropping to a lower energy state and releasing energy as heat to the heat sink (hot side).Thermoelectric can be used to heat and to cool, depending on the direction of the current. In an application requiring both heating and cooling, the design should focus on the cooling mode.

Using a thermoelectric in the heating mode is very efficient because all the internal heating (Joulian heat) and the load from the cold side is pumped to the hot side. This reduces the power needed to achieve the desired heating.

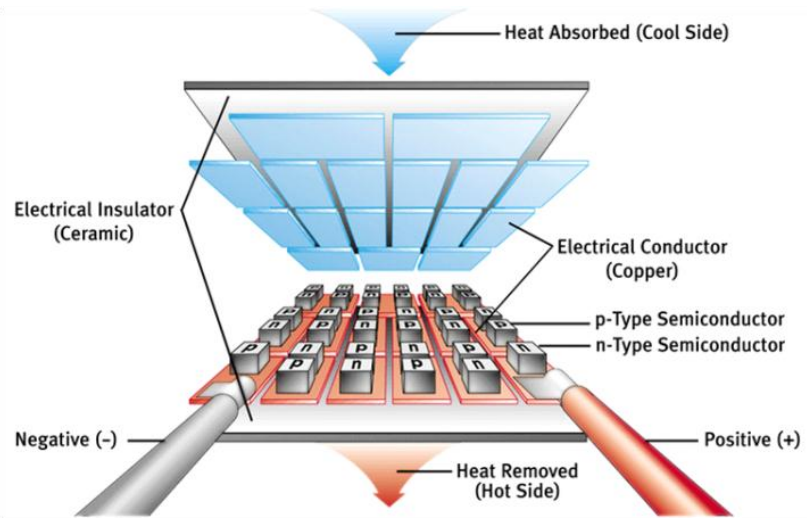

Figure 3.TEC Principle of operation

\section{Thermoelectric materials}

Solid-state materials are generally classified by their electrical properties as metals, semiconductors, and insulators. The useful crystalline materials for thermoelectric elements are classified as semiconductors, having an electrical conductivity between metals and insulators. With respect to thermoelectric materials, the most important material characteristic- the figure of merit ( $\mathrm{Z}$ ) describes the usefulness of a material by the following relationship

$z=\frac{\alpha^{2} \delta}{k}$

Where $\alpha=$ Seebeck coefficient

$\delta=$ electrical conductivity

$k=$ thermal conductivity

The power factor, device efficiency determines the usefulness of a material in thermoelectric systems. These are again determined by the material's electrical
\& thermal conductivity, Seebeck coefficient and behaviour under changing temperatures. For good efficiency materials with high electrical conductivity, low thermal conductivity and high Seebeck coefficient are needed. Due to the small Seebeck coefficient metals have a very limited performance and the main materials of interest are Semiconductors. Pure metals are not good due to their high thermal conductivity, while insulating materials are not good due to their low electrical conductivity.

The thermoelectric semiconductor material most often used in today's TE coolers is an alloy of Bismuth Telluride $\left(\mathrm{Bi}_{2} \mathrm{Te}_{3}\right)$ that has been suitably doped to provide individual blocks or elements having distinct $\mathrm{N}$ and $\mathrm{P}$ characteristics. Other thermoelectric materials include Lead Telluride (PbTe), Silicon Germanium (SiGe), and Bismuth-Antimony (Bi-Sb) alloys, which may be used in specific situations; however, Bismuth Telluride is the best material in most computer cooling scenarios.

Bismuth Telluride has two important characteristics. Due to its crystal structure, it is highly anisotropic. Its electrical resistance is about four times greater parallel to the axis of crystal growth than perpendicular to it. Thermal conductivity, on the other hand, is about double parallel to the crystal-growth axis than perpendicular direction. Hence the anisotropic behavior of resistance is greater than that of thermal conductivity, and the highest Figure-ofMerit occurs in the parallel orientation. The thermoelectric elements must be incorporated into a cooling module so that the crystal growth axis is parallel to the length of each element (perpendicular to the ceramic plates), so that this anisotropy is harnessed for optimum cooling.

Another interesting characteristic of Bismuth Telluride is that its crystals are made up of hexagonal layers of similar atoms. While alternate layers of Bismuth and Tellurium are held together by strong covalent bonds, adjacent layers of Tellurium are held together only by weak vander Waals bonds. As a result, crystalline Bismuth Telluride cleaves readily along these Tellurium-Tellurium layers (like Mica sheets) Fortunately the cleavage planes generally run parallel to the C-axis, so the material is quite strong when assembled into a thermoelectric cooling module.

Thus Commonly used thermoelectric materials are Bismuth Telluride $\left(\mathrm{Bi}_{2} \mathrm{Te}_{3}\right)$, Lead Telluride (PbTe),Silicon Germanium (SiGe) and Cobalt Antimony $\left(\mathrm{CoSb}_{3}\right)$, among which $\mathrm{Bi}_{2} \mathrm{Te} 3$ is the most commonlyused one. These materials usually process a $Z T$ value (figure of merit at temperature) less than one. From 1960s to 1990s, developments in materials in the view of increasing $Z T$ value were modest, but after the mid-1990s, by using nano structural engineering thermoelectric material efficiency is greatly improved. Thermoelectric materials such as skutterudites, clathrates and half-Heusler alloys, which are principally produced through doping method are developed but not exploited for commercial use Dongliang et al. 


\section{Recent Developments in Thermoelectric Refrigeration and Air Conditioning Systems}

The thermoelectric refrigeration system powered by solar cells generated DC voltage was designed and developed byDai et al. They also carried out experimental investigation and analysis. They developed a prototype which consists of a thermoelectric module, array of solar cell, controller, storage battery and rectifier. The system with solar cells and thermoelectric refrigerator is used for outside purpose in daytime and system with storage battery, $\mathrm{AC}$ rectifier and TER is used in night time when $\mathrm{AC}$ power is available. Experimental analysis on the unit was conducted mainly under sunshine conditions. The studied refrigerator can maintain the temperature in refrigerated space at $5-10^{\circ} \mathrm{C}$, and has a COP about 0.3 under given conditions. A prototypeof thermoelectric domestic-refrigerators with different heat exchanger combinationwas developed by Min et al. They evaluated their cooling performances in terms of the COP, heat pumping capacity, cooling down rate and temperature stability. The COP of a thermoelectric refrigerator is found to be 0.3-0.5 for a typical operating temperature of $5^{\circ} \mathrm{C}$ with ambient at $25^{\circ} \mathrm{C}$. The potential improvement in the cooling performance of a thermoelectric refrigerator is also investigated employing a realistic model, with experimental data obtained from this work. The results show that an increase in its COP is possible through improvements in module contact resistances, thermal interfaces and the effectiveness of heat exchangers. Vian et al. shows the development of a thermo-siphon with phase change (TSF) which improvesthe thermal resistance of the heat exchanger of the hot side of the Peltier pellet by $36 \%$, what producesan increase in the COP of a domestic thermoelectric refrigerator of $26 \%$ at an ambient temperature of $20^{\circ} \mathrm{C}$, and $36.5 \%$ at $30^{\circ} \mathrm{C}$. Along this line, Riffat et al. apply the thermo-siphon system, in athermoelectric heat pump system that works as cooling and heating mode. Putra et al. designed, manufactured and tested a portable vaccine carrier box employing thermoelectric module and heat pipe. The position of heat pipe as a heat sink on the hot side of the TEM enhanced the cooling performance. The minimum temperature in the vaccine carrier cabin reached $-10^{\circ} \mathrm{C}$, which shows that vaccine carrier can store the vaccine at desired temperature. Adeyanju et al. carried out a theoretical and experimental analysis of a thermoelectric beverage chiller. Comparison were also made between the thermoelectric beverage chiller's cooling time with cooling times obtained from the freezer space and cold space of a household refrigerator. The result shows that for the refrigerator freezer space, the temperature of the water decreased linearly with increasing time and for thermoelectric beverage chiller the temperature of the water decreased exponentially with increasing time. Lertsatitthanakorn et al. evaluated the cooling performance and thermal comfort of a thermoelectric ceiling cooling panel (TE-CCP) system composed of 36 TEM. The cold side of the TEM was fixed to an aluminum ceiling panel to cool a test chamber of $4.5 \mathrm{~m}^{3}$ volume, while a copper heat exchanger with circulating cooling water at the hot side of the TE modules was used for heat release. Thermal acceptability assessment was performed to find out whether the indoor environment met the ASHRAE Standard-55's $80 \%$ acceptability criteria. The standard was met with the TE-CCP system operating at $1 \mathrm{~A}$ of current flow with a corresponding cooling capacity of $201.6 \mathrm{~W}$, which gives the COP of 0.82 with an average indoor temperature of $27 \mathrm{oC}$ and $0.8 \mathrm{~m} / \mathrm{s}$ indoor air velocity. Gillott et al. conducted an experimental investigation of thermoelectric cooling devices for small-scale space conditioning applications in buildings. They performed a theoretical study to find the optimum operating conditions, which were then applied in the laboratory testing work. A TEC unit was assembled and tested under laboratory conditions. Eight pieces of Ultra TEC were shown to generate up to $220 \mathrm{~W}$ of cooling effect with a COP of 0.46 under the input current of $4.8 \mathrm{~A}$ for each module. Bansal et al. conducted a detail study on energy efficiency and cost-effectiveness for vapour compression, thermoelectric and absorption refrigeration of similar capacity (about 50 litre). The investigated result show that vapour compression refrigerator was the most energy efficient (with a COP of 2.59) followed by thermoelectric (COP of 0.69) and absorption refrigerator (COP of 0.47). The Cost analysis results show that the total purchasing and operating costs over the life of the systems was the lowest for the vapour-compression unit at NZ\$506, followed by the thermoelectric (\$1381.2) and the absorption refrigerator $(\$ 1387.4)$. The researchers finally concluded that the VC refrigerator was the most energy efficient and cheaper unit followed by the thermoelectric and the absorption refrigeration.

\section{Conclusions}

Thus thermoelectric modules offer many advantages including: No moving parts, Small and lightweight, Maintenance-free Acoustically silent and electrically quiet, Heat or cool by changing direction of current, flow Wide operating temperature range, Highly precise temperature control (to within $0.1^{\circ} \mathrm{C}$ ), Operation in any orientation, zero gravity and high G- levels Environmentally friendly and Sub-ambient cooling. The paper focuses on thermoelectric device principles and theory, thermoelectric Principle of Operation, thermoelectric materials as well as recentdevelopments in thermoelectric Refrigeration and Air Conditioning Systems.

\section{References}

Refrigeration and air conditioning, Lesson 8 Methods of producing Low Temperatures, IIT Kharagpur.

Faculty of engineering Electronics Cooling Methods in Industry, CAIRO University, higher education enhancement project fund. 
Dongliang Zhao, Gang Tan, (February 2014), A review of thermoelectric cooling: Materials, modeling and applications, Applied Thermal Engineering, Published by Elsevier Ltd.

Manoj Kumar Rawat(2013) et.al,A review on developments of thermoelectric Refrigeration and air conditioning systems: a novel Potential green refrigeration and air conditioning Technology. International Journal of Emerging Technology and Advanced EngineeringVolume 3, Special Issue 3: ICERTSD,pages 362-367

Edward S. Kolesar, Jr., Captain USAF, December (1981), Aeromedical reviewThermoelectric cooling:Review and application,usaf school of aerospace medicinedivision (AFSC)brooks air force base, texas 78235

H. E. Lenz, UebereinigeVersucheimGebiete des Galvanismus, St. Pétersb. Acaf. Sci. Bull, vol. III, pp. 321$326,1838$.

E. Altenkirch, Uber den nutzeffekt der thermosaulephysikalischezeitschrift, vol. 10, p. 560, 1909.

A. F. Loffe, Semiconductor thermoelements\& thermoelectric cooling, Infosearch, 1957

D. M. Rowe and C. M. Bhandari, Modern Thermoelectrics, Hot Technology, 1983

H. J. Goldsmid and R. W. Douglas, The use of semiconductors in thermoelectric refrogeration, Br. J. Applied physics, vol. 5, no. 11, p. 386, 1954.

Dai Y. J., Wang R. Z. and L. Ni, 2003, Experimental investigation and analysis on a thermoelectric refrigerator driven by solar cells, Solar energy material and solar cells 77:377-391.
Gao Min and Rowe D.M., 2006, Experimental evaluation of prototype thermoelectric domestic-refrigerators, Applied Energy, 83 (2): 133-152.

J. Vián and D. Astrain, Development of a heat exchanger for the cold side of a thermoelectric module, Applied Thermal Engineering, vol. 28, no. 11-12, pp. 1514-1521, August 2008.

S. Riffat and G. Qiu, Comparative investigation of thermoelectric air conditioner versus vapour compression and absorption air conditioners, Applied Thermal Engineering, vol. 24, pp. 1979-1993, 2004.

Putra N., 2009, Design, manufacturing and testing of a portable vaccine carrier box employing thermoelectric module and heat pipe, Journal of Medical Engineering \& Technology, 33 (3): 232-237.

Adeyanju A.A., E. Ekwue and W. Compton, 2010, Experimental and Theoretical Analysis of a Beverage Chiller, Research Journal of Applied Science, 5 (3): 195203.

Lertsatitthanakorn C., LamulWiset, and S. Atthajariyakul, 2009, Evaluation of the Thermal Comfort of a Thermoelectric Ceiling Cooling Panel (TE-CCP), System Journal of Electronic Materials, 38: (7) 1472-1477.

Gillott Mark, Liben Jiang and SaffaRiffat, 2010, An investigation of thermoelectric cooling devices for smallscale space conditioning applications in buildings, International Journal of Energy Research, 34: 776-786. Bansal P. K. and Martin A., 2000, Comparative study of vapour compression, thermoelectric and absorption refrigerators, International Journal of Energy Research, 24 93-107. 